\title{
HESITANT FUZZY LINGUISTIC DNMA METHOD WITH CARDINAL CONSENSUS REACHING PROCESS FOR SHOPPING MALL LOCATION SELECTION
}

\author{
Song NIE ${ }^{1}$, Huchang LIAO (D) 1,2, ${ }^{*}$, Xingli WU ${ }^{1}$, Ming TANG ${ }^{1}$, Abdullah AL-BARAKATI (i) 2 \\ ${ }^{1}$ Business School, Sichuan University, Chengdu 610064, China \\ ${ }^{2}$ Faculty of Computing and Information Technology, King Abdulaziz University, Jeddah 21589, Saudi Arabia
}

Received 25 April 2019; accepted 12 June 2019

\begin{abstract}
The hesitant fuzzy linguistic term set is an effective tool to express qualitative evaluations since it is close to human reasoning and expressing habits. In this paper, we propose a multi-expert multi-criterion decision-making method integrating the double normalization-based multi-aggregation (DNMA) method with a cardinal consensus reaching process, where the assessments of alternatives over multiple criteria are expressed as hesitant fuzzy linguistic term sets. To do so, the DNMA method involving double normalizations and three aggregation tools is extended to deal with the hesitant fuzzy linguistic information and derive the ranking of alternatives with respect to each expert. In addition, a cardinal consensus reaching process is introduced to help experts reach an acceptable consensus level. In other words, the soft consensus is considered in the multi-expert multi-criterion decision-making process. Subsequently, an extended Borda rule is developed to aggregate the subordinate ranks and integrated scores of alternatives, and then deduce the comprehensive ranking of alternatives. A case study is given to illustrate the practicability of the proposed method for selecting the optimal geographical location of a larger-scale shopping mall in the new urbanization for a construction investment agency. The proposed method is compared with other ranking methods to illustrate its advantages.
\end{abstract}

Keywords: multi-expert multi-criterion decision making, hesitant fuzzy linguistic term set, double normalization-based multiple aggregation method, cardinal consensus method, extended Borda rule, shopping mall location selection.

\section{Introduction}

Decision-making problems are usually defined under uncertain and vague conditions in real situations. In many cases, the nature of uncertainty is not probabilistic but imprecise or ambiguous. To describe uncertain cognitions, Zadeh (1965) proposed the fuzzy set that used membership degrees to describe the strength of elements belonging to a set. In a fuzzy set, the membership degree of an element is a single value between zero and one. However, there are some limitations in expressing evaluations by single values. In this regard, Torra (2010) proposed the hesitant fuzzy set characterized by a function that returned a set of membership values for each element in the domain. Given that linguistic terms are closer to the habit of human expressions than quantitative membership degrees, Rodríguez, Martínez, and Herrera (2012) extended the hesitant fuzzy set to the linguistic background and introduced the hesitant fuzzy linguistic term set (HFLTS). To conform to human thinking and inference process,
Rodríguez et al. (2012) provided a way of linguistic elicitation for HFLTSs by using comparative terms based on context-free grammars.

As an effective qualitative evaluation method, many operations on HFLTSs were proposed based on the subscripts of linguistic terms (Rodríguez et al., 2012). Considering that the subscript-based operations cannot deal with unbalanced linguistic term sets (LTSs), Liao et al. (2019) proposed a score function of the HFLTS, which could translate linguistic terms to specific numerical values to simplify the calculation process. Besides, many methods have been proposed for solving the multi-criteria decision-making $(\mathrm{MCDM})$ problems in which the linguistic evaluations are expressed by HFLTSs, such as the hesitant fuzzy linguistic Technique for Order of Preference by Similarity to Ideal Solution (HFL-TOPSIS) method (Beg \& Rashid, 2013), hesitant fuzzy linguistic VlseKriterijumska Optimizacija I Kompromisno Resenje (HFL-VIKOR) method (Liao, Xu, \& Zeng, 2015), hesitant fuzzy linguistic

*Corresponding author. E-mail: liaohuchang@163.com 
MULTIplicative Multi-Objective Optimization by Ratio Analysis (HFL-MULTIMOORA) method (Liao et al., 2019), hesitant fuzzy linguistic Organísation, rangement et Synthèse de données relarionnelles (HFL-ORESTE) method (Liao, Wu, Liang, Xu, \& Herrera, 2018) and hesitant fuzzy linguistic ELimination Et Choix Traduisant la REalité (HFL-ELECTRE) method (Liao, Wu, Mi, \& Herrera, 2019).

However, all the above MCDM methods only used single normalization technique to nondimensionalize evaluation values under different criteria. In this sense, using a predefined normalization method may bias the results when the normalization tool is not suitable. In this regard, Liao and $\mathrm{Wu}$ (2019) proposed a double normalizationbased multi-aggregation (DNMA) method based on two normalization approaches including the target-based linear normalization and target-based vector normalization. However, the original DNMA method is unable to handle the decision matrices composed by HFLTSs. Therefore, the first motivation of this paper is to combine the DNMA method with HFLTSs to solve problems involving qualitative information.

In multi-expert MCDM (MEMCDM) problems, consensus refers to a degree of agreement among all experts with regard to all alternatives under a specific circumstance (Ben-Arieh \& Chen, 2006). Consensus reaching process has been wildly addressed since it can reduce the conflict among experts, increase the participation of experts and enhance the acceptance of decision-making results (Kahraman, Engin, Kabak, \& Kaya, 2009; Xu, 2009). The second motivation of this paper is to present a cardinal consensus reaching process for the HFL-DNMA method to help experts reach an acceptable consensus in dealing with MEMCDM problems. Besides, the third motivation of this paper is to propose an extended Borda rule to integrate the scores and ranks of alternatives concerning multiple experts to obtain a collective ranking set.

Furthermore, it is known that large-scale shopping malls play a leading role in booming the market, stabilizing prices, promoting production, demonstrating civilization and better meeting the needs of people. In the process of new urbanization, how to choose the geographical location of a shopping mall to obtain maximum benefits plays a vital role in the future development for an investment agency. Shopping mall location selection is the first step in the operation of the whole investment and construction project. Once the location is not well chosen, it may greatly increase the risk, leading to the lack of competitiveness of the market and poor operating conditions. In recent years, how to select an appropriate large shopping mall location has become a hot research topic. Many researchers have proposed different methods from different points of view (Cheng, Li, \& Yu, 2005, 2007; Song, Yuan, \& Zhang, 2008; Gundogdu, 2013; Elsamen \& Hiyasat, 2017; Elevli, 2014; Can \& Delice, 2018; Canas, Ferreira, \& Meidutè-Kavaliauskienè, 2015). However, to the best of our knowledge, there are few researches considering both the qualitative and quantitative criteria which are usu- ally existed in the selection of shopping mall locations. Furthermore, language is a flexible and effective way for the evaluations of shopping mall locations compared with fuzzy sets which are only expressed as numerical numbers. In this study, we consider both the qualitative and quantitative criteria for the assessments of alternatives in the process of shopping mall location selections. We use the proposed the HFL-DNBMA method with the cardinal consensus reaching process to solve the MEMCDM problem concerning selecting the optimal geographical location of the shopping mall for a construction investment agency in the new urbanization is provided. Comparative analyses are also given to demonstrate its advantages.

To sum up, the motivation of this paper involves the HFL-DNMA method, a cardinal consensus reaching process, an extended Borda rule, and a case study to illustrate the feasibility of the proposed method. This paper tries to achieve the following theoretical contributions:

1. The DNMA method is extended to the hesitant fuzzy linguistic context considering the hesitant degree and score function of the HFLTS.

2. Based on the subordinate ranks obtained by the HFL-DNMA method, a cardinal consensus reaching process is proposed to help expert group reach an acceptable consensus level.

3. An extended Borda rule is proposed to obtain the final comprehensive ranking of alternatives for multi-expert multi-criterion decision-making problems.

The rest of this paper is organized as follows: Section 1 reviews the concepts related to HFLTSs and the main idea of the DNMA method. Section 2 introduces the HFL-DNMA method, the cardinal consensus reaching process, the extended Borda rule and the procedure of the cardinal consensus-based HFL-DNMA method. Section 3 conducts a case study and comparative analyses with other ranking methods. The paper ends with concluding remarks.

\section{Related works and literature review}

To facilitate our presentation, in this section, we review the concepts related to HFLTSs and the main idea of the DNMA method. We also summarize the primary methods on the selection of shopping mall location as well as the research gap of these methods in qualitative and quantitative conditions. Finally, we introduce the primary approaches of reaching consensus for decision-making problems.

\subsection{Literature review on the hesitant fuzzy linguistic term set}

Rodríguez et al. (2012) introduced the concept of HFLTS. Subsequently, Liao, Xu, Zeng, and Merigó (2015) redefined and formalized the HFLTS mathematically as follows: Let $x \in X$ be fixed and $S=\left\{s_{t} \mid t=-\tau, \cdots, 0, \cdots, \tau\right\}$ be an LTS. An HFLTS on $X, H_{S}$, is in mathematical form of $H_{S}=\left\{<x, h_{S}(x)>\mid x \in X\right\}$, where $h_{S}\left(x_{i}\right)=\left\{s_{\varphi_{l}}\left(x_{i}\right) \mid s_{\varphi_{l}}\left(x_{i}\right) \in S ; l=1, \cdots, L ; \varphi_{l} \in\{-\tau, \cdots,-1,0,1, \cdots, \tau\}\right\}$ 
is a set of continuous values in $S$. For convenience, $h_{S}(x)$ is called the hesitant fuzzy linguistic element (HFLE). It is noted that the discrete LTS can be extended to continuous form, i.e., $S^{\prime}=\left\{s_{t} \mid t=[-\tau, \tau]\right\}$ (Liao, Xu, Herrera-Viedma, \& Herrera, 2018).

Since HFLTSs are not similar to the way of human thinking and reasoning, Rodríguez et al. (2012) introduced the context-free grammar $G_{H}$ to generate linguistic expressions that were much closer to the way of human expressions. Then, the transformation function $E_{G_{H}}: l l \rightarrow h_{S}$ can be used to transform the linguistic expression $l l$ obtained by $G_{H}$ into an HFLE $h_{S}$ (Rodríguez et al., 2012). Concerning various linguistic expressions, there are different types of transformation functions. The most representative ones are:

$E_{G H}\left(s_{t}\right)=\left\{s_{t} \mid s_{t} \in S\right\}$

$E_{G H}$ (at least $\left.s_{m}\right)=\left\{s_{t} \mid s_{t} \in S\right.$ and $\left.s_{t} \geq s_{m}\right\}$;

$E_{G H}$ (between $s_{m}$ and $s_{n}$ ) $=\left\{s_{t} \mid s_{t} \in S\right.$ and $\left.s_{m} \leq s_{t} \leq s_{n}\right\}$.

Based on the linguistic scale function of linguistic terms and considering the hesitancy degree of an HFLE, Liao et al. (2019) proposed a score function to convert the HFLE into a specific numerical value. The score function of the HFLE is represented as follows:

$$
E\left(h_{S}\right)=\left(1-H D\left(h_{S}\right)\right) \times\left(\frac{1}{L} \sum_{l=1}^{L} g\left(s_{\varphi_{l}}\right)\right),
$$

where: $g$ is a linguistic scale function which translates the linguistic terms into their semantics belonging to $[0,1]$. For the balanced LTS, there is $g\left(s_{\alpha}\right)=(\alpha+\tau) / 2 \tau$. For other cases, please refer to Liao et al. (2019). $H D\left(h_{S}\right)$ is a hesitancy function to measure the inherent hesitant information of the HFLE. There is $H D\left(h_{S}\right)=\frac{L \ln L}{(2 \tau+1) \ln (2 \tau+1)}$.

\subsection{Literature review on the DNMA method}

The DNMA method (Liao \& Wu, 2019) employed three different aggregation operators with different functions, which makes the method flexible regarding different decision-making requirements. In addition, the proposed method can be applied to deal with the decision-making problems that contain both qualitative and quantitative criteria. The DNMA method mainly includes three kinds of aggregation functions based on the target-based linear normalization and target-based vector normalization. Let $A=\left\{a_{1}, a_{2}, \cdots, a_{m}\right\}$ be a set of alternatives and $C=\left\{c_{1}, \cdots, c_{n}\right\}$ be a set of criteria with the weight vector $W=\left(\omega_{1}, \omega_{2}, \cdots, \omega_{n}\right)^{T} . X=\left(x_{i j}\right)_{m \times n}$ is a decision-making matrix where $x_{i j}$ is the value of alternative $a_{i}$ on criterion $c_{j}$. In the original DNMA method (Liao \& Wu, 2019), there are three subordinate aggregation models, i.e., the complete compensatory model (CCM), un-compensatory model (UCM) and incomplete compensatory model (ICM).

The CCM is based on the arithmetic weighted aggregation operator, shown as:

$$
u_{1}^{\prime}\left(a_{i}\right)=\sum_{j=1}^{n} \omega_{j} y_{i j}^{1},
$$

where: $y_{i j}^{1}$ is the linear normalization value of $x_{i j}$ with $y_{i j}^{1}=1-\frac{\left|x_{i j}-r_{j}\right|}{\max _{i}\left|x_{i j}-r_{j}\right|}$. Ranking $u_{1}^{\prime}\left(a_{i}\right)$ in descending order, the first type of ranking, $r_{1}^{\prime}\left(a_{i}\right) \quad(i=1,2, \cdots, m)$, is obtained.

The UCM is shown as

$$
u_{2}^{\prime}\left(a_{i}\right)=\max _{j} \omega_{j}\left(1-y_{i j}^{1}\right) .
$$

Ranking $u_{2}^{\prime}\left(a_{i}\right)$ in ascending order, the second type of ranking, $r_{2}^{\prime}\left(a_{i}\right)(i=1,2, \cdots, m)$, is obtained.

Because the linear normalization cannot reflect the quality of original values, the results obtained by the above two aggregation functions would be misguided in some cases. Thus, Liao and $\mathrm{Wu}$ (2019) further proposed the third aggregation function, ICM, which is represented as

$$
u_{3}^{\prime}\left(a_{i}\right)=\prod_{j}\left(y_{i j}^{2}\right)^{\omega_{j}},
$$

where: $y_{i j}^{2}$ is the vector normalization value of $x_{i j}$ with $y_{i j}^{2}=1-\left|x_{i j}-r_{j}\right| / \sqrt{\sum_{i=1}^{m}\left(x_{i j}\right)^{2}+\left(r_{j}\right)^{2}}$. The third type of rank set, $r_{3}^{\prime}\left(a_{i}\right) \quad(i=1,2, \cdots, m)$, could be obtained in descending order of $u_{3}^{\prime}\left(a_{i}\right) \quad(i=1,2, \cdots, m)$.

Finally, the integrated scores are calculated by (Liao \& Wu, 2019):

$$
\begin{aligned}
& S_{i}=\sqrt{\varphi\left(\frac{u_{1}^{\prime}\left(a_{i}\right)}{\sqrt{\sum_{i=1}^{m}\left(u_{1}^{\prime}\left(a_{i}\right)\right)^{2}}}\right)^{2}+(1-\varphi)\left(\frac{m-r_{1}^{\prime}\left(a_{i}\right)+1}{m(m+1) / 2}\right)^{2}}- \\
& \sqrt{\varphi\left(\frac{u_{2}^{\prime}\left(a_{i}\right)}{\sqrt{\sum_{i=1}^{m}\left(u_{2}^{\prime}\left(a_{i}\right)\right)^{2}}}\right)^{2}+(1-\varphi)\left(\frac{r_{2}^{\prime}\left(a_{i}\right)}{m(m+1) / 2}\right)^{2}}+ \\
& \sqrt{\varphi\left(\frac{u_{3}^{\prime}\left(a_{i}\right)}{\sqrt{\sum_{i=1}^{m}\left(u_{3}^{\prime}\left(a_{i}\right)\right)^{2}}}\right)^{2}+(1-\varphi)\left(\frac{m-r_{3}^{\prime}\left(a_{i}\right)+1}{m(m+1) / 2}\right)^{2}},
\end{aligned}
$$

where: $\varphi$ is a coefficient to emphasize the significance between the subordinate ranks and utility values.

Without lose of generality, $\varphi$ can be 0.5 . The final rank set $R=\left\{r\left(a_{1}\right), \cdots, r\left(a_{m}\right)\right\}$ of alternatives is obtained in the descending order of $S_{i}(i=1,2, \cdots, m)$.

\subsection{Literature review on the selection of shopping mall location}

Shopping malls play a vital role in a city. In recent years, the commercial real estate has become a hot investment. 
Selecting an appropriate shopping mall location becomes one of the research objects. Cheng et al. (2005) employed the analytic network process (ANP) with a comprehensive analytic framework to select the optimal location for a shopping mall. Subsequently, Cheng et al. (2007) introduced a geographical information system (GIS) method to select shopping mall location, in which the electronic mapping technology was used to construct interactive multi-layer maps. Song et al. (2008) combined the analytic hierarchy process (AHP) and multi-level fuzzy comprehensive evaluation to obtain a good shopping mall location. Based on the GIS, Gundogdu (2013) proposed a decision-making process to select the best shopping mall location on a regional basis by loading semantic data about geographical data, in which economic and social data, population density, quality of life and geographical data were considered. Similarly, based on the ArcGIS's network analysis tool and time-resistance approach, Elsamen and Hiyasat (2017) examined the optimal shopping mall location selections in the area of west Amman in Jordan. Considering the vagueness of subjective evaluations, Elevli (2014) suggested the fuzzy PROMETHEE (preference ranking organization method for enrichment evaluation) method to select the optimal potential logistics center locations where fuzzy sets were used to portray the uncertain assessments. Can and Delice (2018) studied a task-based fuzzy integrated MCDM approach for the shopping mall selection considering universal design criteria. For the MCDM problem, Canas et al. (2015) presented a methodological proposal with multiple criteria decision analysis to obtain the optimal selection.

Shopping malls are a classification of commercial real estate. As the first step of whole project investment, the location selection plays an important role. At present, the situation of commercial real estate location in China is that practice is far ahead of theory, which greatly increases the risk of investment.

In the aforementioned methods on the selection of shopping mall, they are suitable to solve the decisionmaking problems for single qualitative or quantitative conditions. However, many decision-making problems on the selection of shopping mall are involved with qualitative and quantitative condition. Therefore, it is necessary to establish an effective index system of shopping mall location and study it with modern decision-making theory.

\subsection{Literature review on the approaches of consensus in decision-making problems}

Consensus process can provide the feedback for experts and improve the acceptance of final decision-making results. Therefore, consensus has been widely discussed. Many approaches have been investigated to improve the consensus levels of experts (Kahraman et al., 2009; Parreiras, Ekel Martini, \& Palhares, 2010). A common process of the consensus-based group decision-making (GDM) methods (Herrera, Herrera-Viedma, \& Verdegay, 1996; $\mathrm{Xu}, 2005)$ is to measure the consensus of individual evalua- tions, and then aggregates the individual decision matrices with a high consensus degree into a group decision matrix. Perez, Cabrerizo, Alonso, and Herrera-Viedma (2014) proposed a consensus model for heterogeneous GDM problems that were guided by the heterogeneity criterion. A trust induced recommendation mechanism for reaching consensus in GDM problems (Liu, Liang, Chiclana, \& $\mathrm{Wu}, 2017$ ) was introduced to generate personalized advice for low-consensus experts to reach a high-level consensus. Dong, Zhang, Hong, and Xu (2010) introduced a cardinal consensus by measuring the distance between individual preference values and collective preference values.

Based on individual preference values and collective preference values, the preferences of experts on alternatives can be easily shown. Subsequently, we shall present a simple cardinal consensus reaching process based on the HFL-DNMA method, which can provide feedback for experts to reach an acceptable consensus in dealing with MEMCDM problems.

\section{The cardinal consensus-based HFL-DNMA method}

In this section, we extend the original DNMA method to the hesitant fuzzy linguistic environment to solve MEM$\mathrm{CDM}$ problems and consider the consensus degree of multiple experts. Besides, the cardinal consensus reaching process is introduced. Then, an extended Borda rule is introduced to obtain the final comprehensive ranking of alternatives.

\subsection{The HFL-DNMA method}

An MEMCDM problem contains a finite set of alternatives, $A=\left\{a_{1}, a_{2}, \cdots, a_{m}\right\}(m \geq 2)$, and a set of experts, $q=\left\{e_{1}, e_{2}, \cdots, e_{Q}\right\}(Q \geq 2)$. Each alternative is measured by a finite set of criteria $C=\left\{c_{1}, c_{2}, \cdots, c_{n}\right\}(n \geq 2)$ with the weight vector $W=\left(\omega_{1}, \omega_{2}, \cdots, \omega_{n}\right)^{T}$ which satisfies $0 \leq \omega_{j} \leq 1$ and $\sum_{j}^{n} \omega_{j}=1$. By integrating the original DNMA method with HFLEs, the HFL-DNMA method can be proposed to deal with MEMCDM problems with both quantitative and qualitative information. We present the procedure of the HFL-DNMA method as follows:

Step 1. Determine alternatives $a_{i}(i=1,2, \cdots, m)$, criteria $c_{j}(j=1,2, \cdots, n)$ and invite experts to make assessments. The criteria are classified into three categories, i.e., cost, benefit and target-based criteria. Assume that there are $z$ qualitative criteria $\left(c_{1}, c_{2}, \cdots, c_{z}\right)$ and $n-z$ quantitative criteria $\left(c_{z+1}, c_{z+2}, \cdots, c_{n}\right)$.

Step 2. Collect the linguistic assessments of experts concerning the alternatives on qualitative criteria. We use the context-free grammar $G_{H}$ and the transformation function $E_{G_{H}}$ to get the HFLEs $h_{S}^{(q) i j}$ $(i=1,2, \cdots, m ; j=1,2, \cdots, z ; q=1,2, \cdots, Q)$. For the quantitative criteria, the values of alternatives are represented as numerical values $x^{i j(q)}(i=1,2, \cdots, m ; j=z, z+1, \cdots, n)$. Then, the individual decision matrix corresponding to expert $e_{q}$ 
can be established by all $h_{S}^{i j(q)}(i=1,2, \cdots, m ; j=1,2, \cdots, z)$ and $x^{i j(q)}(i=1,2, \cdots, m ; j=z, z+1, \cdots, n)$, which can be denoted as

$$
\begin{aligned}
& D^{(q)}=\left[\begin{array}{llllll}
h_{S}^{11(q)} & \cdots & h_{S}^{1 z(q)} & x^{1 z+1(q)} & \cdots & x^{1 n(q)} \\
\vdots & \ddots & \vdots & \vdots & \ddots & \vdots \\
h_{S}^{i 1(q)} & \cdots & h_{S}^{i z(q)} & x^{i z+1(q)} & \cdots & x^{i n(q)} \\
\vdots & \ddots & \vdots & \vdots & \ddots & \vdots \\
h_{S}^{m 1(q)} & \cdots & h_{S}^{m z(q)} & x^{m z+1(q)} & \cdots & x^{m n(q)}
\end{array}\right], \\
& q=1,2, \cdots, Q .
\end{aligned}
$$

Step 3. To reduce the complexity of HFLEs during the process of calculations, by Eq. (1), the HFLE $h_{S}^{i j(q)}$ in the decision-making matrix $D^{(q)}$ is transformed into its score, $E\left(h_{S}^{i j(q)}\right)$. Then, we have

$X^{(q)}=\left[\begin{array}{llllll}E\left(h_{S}^{11(q)}\right) & \cdots & E\left(h_{S}^{1 z(q)}\right) & x^{1 z+1(q)} & \cdots & x^{1 n(q)} \\ \vdots & \ddots & \vdots & \vdots & \ddots & \vdots \\ E\left(h_{S}^{i 1(q)}\right) & \cdots & E\left(h_{S}^{i z(q)}\right) & x^{i z+1(q)} & \cdots & x^{i n(q)} \\ \vdots & \ddots & \vdots & \vdots & \ddots & \vdots \\ E\left(h_{S}^{m 1(q)}\right) & \cdots & E\left(h_{S}^{m z(q)}\right) & x^{m z+1(q)} & \cdots & x^{m n(q)}\end{array}\right]$, $q=1,2, \cdots, Q$.

Step 4. Calculate target-based linear and vector normalized values for each transformed matrix $X^{(q)}$. Motivated by the target-based linear normalized formula proposed in Liao and $\mathrm{Wu}$ (2019), the hesitant fuzzy linguistic target-based linear normalized formula is shown as follows:

$$
\kappa_{i j(q)}^{1}=\left\{\begin{array}{cl}
1-\frac{\left|E\left(h_{S}^{i j(q)}\right)-\rho_{j(q)}\right|}{\max _{i}\left|E\left(h_{S}^{i j(q)}\right)-\rho_{j(q)}\right|}, & \text { if } j=1,2, \cdots, z \\
1-\frac{\left|x^{i j(q)}-r_{j(q)}\right|}{\max _{i}\left|x^{i j(q)}-r_{j(q)}\right|}, & \text { if } j=z+1, z+2, \cdots, n
\end{array},\right.
$$

where: $\rho_{j(q)}$ is the target value of the qualitative criterion $c_{j}$, for $j=1,2, \cdots, z$, and $r_{j(q)}$ is the target value of the qualitative criterion $c_{j}$ for $j=z, z+1, \cdots, n$ corresponding to expert $e_{q}$. Especially, if $c_{j}$ is in benefit type, $\rho_{j(q)}=\max _{i}\left(E\left(h_{S}^{i j(q)}\right)\right)$ and $r_{j(q)}=\max _{i}\left(x^{i j(q)}\right)$.

In analogous, the hesitant fuzzy linguistic target-based vector normalized formula is shown as follows:

$$
\kappa_{i j(q)}^{2}=\left\{\begin{array}{cl}
1-\frac{\left|E\left(h_{S}^{i j(q)}\right)-\rho_{j(q)}\right|}{\sqrt{\sum_{i=1}^{m}\left(E\left(h_{S}^{i j(q)}\right)\right)^{2}+\left(\rho_{j(q)}\right)^{2}}}, \quad \text { if } j=1,2, \cdots, z \\
1-\frac{\left|x^{i j(q)}-r_{j(q)}\right|}{\sqrt{\sum_{i=1}^{m}\left(x^{i j(q)}\right)^{2}+\left(r_{j(q)}\right)^{2}},} \quad \text { if } j=z+1, z+2, \cdots, n
\end{array} .\right.
$$

Step 5. Calculate the utility values $u_{1}^{(q)}\left(a_{i}\right), u_{2}^{(q)}\left(a_{i}\right)$ and $u_{3}^{(q)}\left(a_{i}\right)(i=1,2, \cdots, m)$ for different alternatives. Then, according to these utility values, we obtain three types of subordinate ranks $r_{1}^{(q)}\left(a_{i}\right), r_{2}^{(q)}\left(a_{i}\right)$ and $r_{3}^{(q)}\left(a_{i}\right)$ $(i=1,2, \cdots, m)$, respectively.

$$
\begin{aligned}
& u_{1}^{(q)}\left(a_{i}\right)=\sum_{j=1}^{n} \omega_{j} \kappa_{i j(q)}^{1} ; \\
& u_{2}^{(q)}\left(a_{i}\right)=\max _{j} \omega_{j}\left(1-\kappa_{i j(q)}^{1}\right) ; \\
& u_{3}^{(q)}\left(a_{i}\right)=\prod_{j}\left(\kappa_{i j(q)}^{2}\right)^{\omega_{j}} .
\end{aligned}
$$

Step 6. Normalize the subordinate utility values $u_{1}^{(q)}\left(a_{i}\right), u_{2}^{(q)}\left(a_{i}\right)$ and $u_{3}^{(q)}\left(a_{i}\right)$ by

$$
u_{y}^{N(q)}\left(a_{i}\right)=\frac{u_{y}^{(q)}\left(a_{i}\right)}{\sqrt{\sum_{i=1}^{m}\left(u_{y}^{(q)}\left(a_{i}\right)\right)^{2}}}, y=1,2,3 .
$$

Step 7. Integrate the subordinate ranks and subordinate normalized utility values by Eq. (5), and obtain the integrated scores of all alternatives corresponding to each expert. Then, we can obtain the final rank set of alternatives with respect to each expert as $R^{(q)}=\left\{r^{(q)}\left(a_{1}\right), \cdots, r^{(q)}\left(a_{i}\right), \cdots, r^{(q)}\left(a_{m}\right)\right\}$.

It is a common phenomenon that there are some differences on the integrated scores or subordinate ranks of alternatives with respect to different experts. This is mainly because different experts have different expertise and experience. In this sense, it is necessary to consider whether all experts reach a consensus degree after obtaining the integrated scores and ranks of alternatives. The consensus degree of an expert group in MEMCDM problems is considered to reduce subjective judgments as much as possible. In the next section, we propose a cardinal consensus measure based on the rank sets of alternatives derived by the HFL-DNMA method to check the consensus degree of experts and then develop a consensus reaching process for hesitant fuzzy linguistic MEMCDM problems.

\subsection{A cardinal consensus reaching process for hesitant fuzzy linguistic MEMCDM problems}

The MEMCDM problems have been widely studied, and consensus is an important issue to guarantee that the group members agree to support a decision to obtain the best interest or common goal of the whole group (Lee, 2002; Li, Dong, Herrera, \& Herrera-Viedma, 2017; Wu, Dai, Chiclana, Fujita, \& Herrera-Viedma, 2018). In general, consensus can be divided into two categories, i.e., the hard consensus and soft consensus (Herrera-Viedma, Martínez, Mata, \& Chiclana, 2005). The consensus degree can be represented by " 0 " or " 1 " where " 0 " denotes there is no agreement and " 1 " denotes a full agreement among all experts (Wibowo \& Deng, 2013). In real decision-making process, it is difficult or even impossible for all experts to reach a full agreement. In this regard, the soft consensus 
can be defined as an acceptable solution that "most" experts agree on the result for an MEMCDM problem, even if the solution is not everyone's "favorite". The soft consensus enables experts to provide their assessments in a flexible manner (Ross \& Jayaraman, 2008). Therefore, the soft consensus is appropriate to deal with MEMCDM problems in real situations. In this section, we conduct the cardinal consensus reaching process for hesitant fuzzy linguistic MEMCDM problems where the cardinal consensus is a soft consensus.

Let $R^{(q)}=\left\{r^{(q)}\left(a_{1}\right), r^{(q)}\left(a_{2}\right), \cdots, r^{(q)}\left(a_{i}\right), \cdots, r^{(q)}\left(a_{m}\right)\right\}$ be the rank set of alternatives derived by the HFL-DNMA method based on the linguistic assessments of expert $e_{q}$. The individual preference degree of the rank $r^{(q)}\left(a_{i}\right)$ is defined as Eq. (12). Then, the rank set of expert $e_{q}$ can be transited to the preference set $\eta^{(q)}=\left\{\eta_{1}^{(q)}, \eta_{2}^{(q)}, \cdots, \eta_{m}^{(q)}\right\}$.

$$
\eta_{i}^{(q)}=\left(m-r_{i}^{(q)}\right) / \sum_{i=1}^{m-1} i
$$

Based on the weighted averaging operator, the group preference degree of alternative $a_{i}$ can be calculated by

$$
\overline{\eta_{i}}=\frac{1}{Q} \sum_{q=1}^{Q} \eta_{i}^{(q)}
$$

The Euclidean distance formula is applied to calculate the overall distance between the individual preference degrees corresponding to expert $e_{q}$ and the group preference degree, which is denoted as

$$
d_{E u c}^{(q)}=\sqrt{\frac{1}{m} \sum_{i=1}^{m}\left(\eta_{i}^{(q)}-\overline{\eta_{i}}\right)^{2}} .
$$

Then, the cardinal consensus index $C C I^{(q)}$ of expert $e_{q}$ is defined as:

$$
C C I^{(q)}=1-d_{\text {Euc }}^{(q)} \text {. }
$$

The greater the value of $C C I^{(q)}$ is, the higher the consensus degree of expert $e_{q}$ is. If $C C I^{(q)}=1$, the expert $e_{q}$ has a full cardinal consensus with the expert group. In most cases, the consensus degree does not reach to 1 . In these cases, we need to set an acceptable consensus threshold $\gamma$ and check whether the calculated cardinal consensus index $C C I^{(q)}$ reaches the threshold or not. If yes, then we do not need to implement the consensus reaching process; if not, we need to develop some mechanisms to reach the group consensus. If the decision-making problem is vital for the whole group or the number of experts is small, a large consensus threshold should be set; if the decision-making problem is urgent and time is limited or there are a large number of experts, a related small consensus is acceptable (Xu, Zhong, Chen, \& Zhou, 2015).

Common adjustment strategies include the automatic optimization method and feedback optimization method (Wu \& Xu, 2018). The automatic optimization method does not interact with experts, while the feedback optimization method offers advice to experts, allowing them to make modifications on their linguistic assessments. The advantage of the automatic optimization method is time-saving. The feedback optimization method can communicate with experts, thereby overcoming the limitation that the consensus results are obtained by only calculating numerical values. If time permits, the feedback strategy is better than the automatic optimization method. In this section, we use the feedback mechanism to achieve an acceptable consensus.

The feedback strategy (Wu \& Xu, 2018) mainly includes two rules: identification rules and direction rules. Identification rules are used to identify the experts and alternatives that contribute less to achieve a high-level consensus. Here, we only modify the linguistic assessments of the experts who do not reach the acceptable consensus, while the linguistic assessments of other experts remain unchanged.

- Identification Rule 1. It is used to identify the experts who do not reach the predefined consensus threshold $\gamma$, which can be denoted as

$$
E^{\prime}=\left\{C C I^{(q)} \mid C C I^{(q)}<\gamma\right\} .
$$

- Identification Rule 2. It is used to identify the alternatives that should be modified for the expert $e_{q}$ with $C C I^{(q)}<\gamma$, which can be denoted as

$$
i^{*}=\arg \max _{i=1,2, \cdots, m}\left|\eta_{i}^{(q)}-\overline{\eta_{i}}\right| \text {. }
$$

- Direction Rule 1. For the obtained maximum distance $d_{i^{*}}$ in which $d_{i^{*}}=\eta_{i^{*}}^{(q)}-\overline{\eta_{i^{*}}}<0$, the corresponding expert $e_{q}$ should improve their linguistic preference expressions for the alternative $a_{i}$ as follows: for benefit criteria, the expert can increase their linguistic assessments, and for cost criteria, the expert can decrease their linguistic assessments.

- Direction Rule 2. For the obtained maximum distance $d_{i^{*}}$ in which $d_{i^{*}}=\eta_{i^{*}}^{(q)}-\overline{\eta_{i^{*}}}>0$, the corresponding expert $e_{q}$ should improve their linguistic preference expressions for the alternative $a_{i *}$ as follows: for benefit criteria, the expert can decrease their linguistic assessments, and for cost criteria, the expert can reduce their linguistic assessments.

According to the above direction rules, the expert can change the linguistic assessments of the alternative $a_{i}$ on one or more criteria. Note that there may be more than one alternative which attain the same maximum distance. Under this situation, the alternative can be randomly chosen one from them.

After modifying the linguistic assessments on the alternative $a_{i^{*}}$, if the expert $e_{q}$ reaches the acceptable consensus, the feedback mechanism ends. If not, it is necessary to continue to iterate with feedback mechanism until the expert $e_{q}$ reaches the acceptable consensus. Then, the integrated scores and subordinate ranks of the alternatives for the expert $e_{q}$ should be recalculated by the HFL-DNMA method.

\subsection{The extended Borda rule}

When all experts reach the acceptable consensus, we need to integrate the rankings with respect to all experts into 
a collective one. In this section, we propose an extended Borda rule to integrate the scores and ranks of alternatives with respect to expert $e_{q}$, which can be represented by the following formula:

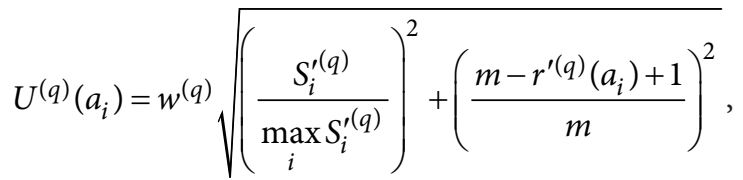

where: $w^{(q)}$ is the weight of the $q$ th expert; $S_{i}^{(q)}$ is the integrated score of alternative $a_{i}$ with respect to the $q$ th expert when all experts reach the acceptable consensus; $r^{\prime(q)}\left(a_{i}\right)$ is the rank of alternative $a_{i}$ determined in descending order of $S_{i}^{\prime(q)}$.

Finally, the comprehensive utility $C U\left(a_{i}\right)$ of alternative $a_{i}$ is obtained by the weight average operator, which is represented as follows:

$$
C U\left(a_{i}\right)=\sum_{q=1}^{Q} U^{(q)}\left(a_{i}\right) .
$$

Based on $C U\left(a_{i}\right)$, the optimal alternative can be selected.

\subsection{The procedure of the cardinal consensus-based HFL-DNMA method}

For the facility of understanding and application, we present the procedure of the cardinal consensus-based HFLDNMA method as follows:

Step 1. For an MEMCDM problem, the linguistic assessments of qualitative criteria are collected from the expert $e_{q}$. These linguistic assessments are transformed into HFLEs by the transformation function $E_{G_{H}}$. The evaluations of quantitative criteria are directly denoted by crisp values. Next, an individual decision-making matrix $D^{(q)}$ including qualitative and quantitative information for the expert $e_{q}$ can be obtained.
Step 2. Based the score function of HFLEs given as Eq. (1), the individual decision-making matrix $D^{(q)}$ can be transformed to the matrix $X^{(q)}$ with crisp values. Next, the normalized values of alternatives are calculated by two target-based normalization formulas given as Eqs (6) and (7).

Step 3. The three subordinate utility values are calculated by Eqs (8)-(10) and further normalized by Eq. (11). Next, the integrated scores and subordinate ranks of all alternatives can be obtained.

Step 4. By Eq. (12), the individual preference degree $\eta_{i}^{(q)}$ of expert $e_{q}$ for alternative $a_{i}$ is calculated based on the subordinate rank $r_{i}^{(q)}$. Then, the collective preference vector $\bar{\eta}_{i}$ can be obtained by Eq. (13). Besides, the overall distance between the individual preference degrees corresponding to expert $e_{q}$ and the group preference degree can be calculated by Eq. (14). Next, the cardinal consensus index $C C I^{(q)}$ of expert $e_{q}$ is calculated by Eq. (15).

Step 5. If $C C I^{(q)}>\gamma$, all experts reach the acceptable consensus. If not, we need to give feedback by Eq. (17) to the expert $e_{q}$ who contributes less to the consensus degree identified by Eq. (16) until the acceptable consensus is reached. For other experts, linguistic assessments remain unchanged. Next, the integrated scores and subordinate ranks for the modified linguistic assessments of expert $e_{q}$ should be recalculated by Steps 1-3.

Step 6. Employing the extended Borda rule given as Eq. (18) to aggregate the integrated scores and subordinate ranks of alternatives after all experts reaching the acceptable consensus. Then, the comprehensive utilities of all alternatives can be obtained. Based on the comprehensive utilities, the collective ranks of alternatives are obtained and the optimal alternative is selected.

In the process of the cardinal consensus-based HFLDNMA method, Step 1 is used to obtain the linguistic assessments and establish the individual decision-making matrix $D^{(q)}$ with transformed HFLEs. Step 2 is to obtain the numerical decision-making matrix $X^{(q)}$ and calculate the normalized values by two target-based normalization

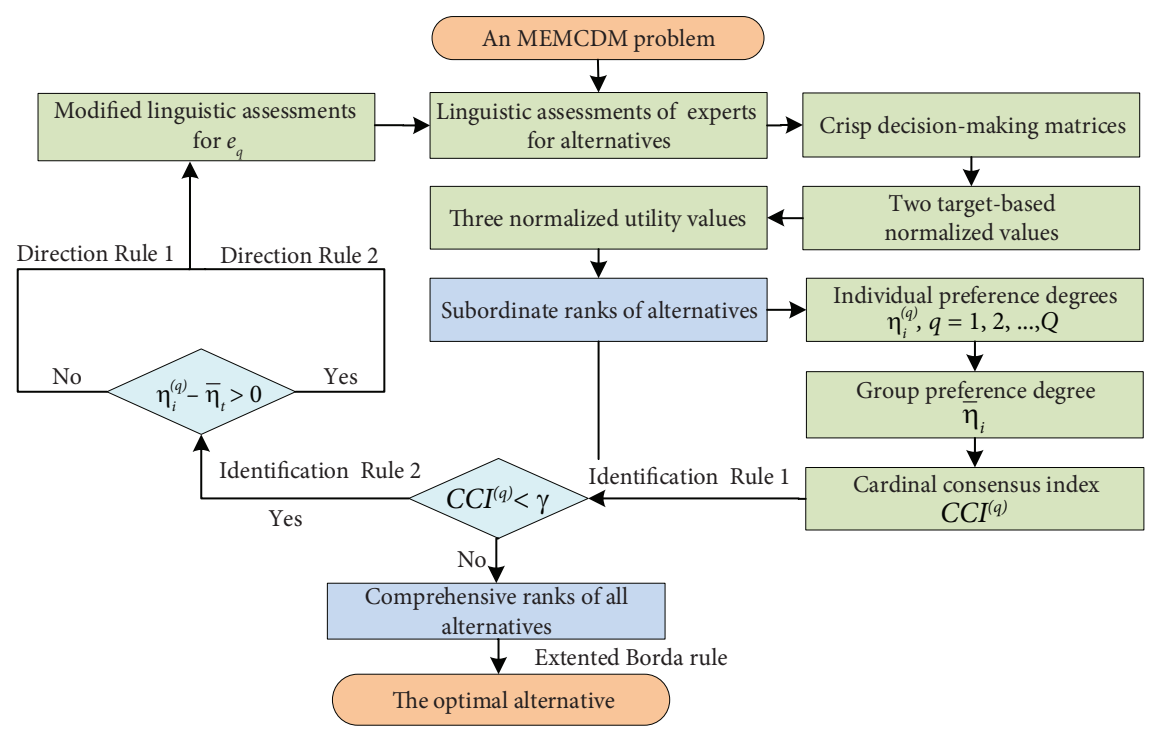

Figure 1. The procedure of the cardinal consensus-based HFL-DNMA method 
formulas. Step 3 is to calculate and normalize the three types of subordinate utility values. Then, the integrated scores and subordinate ranks of alternatives can be obtained. Step 4 is to calculate the individual preference degree $\eta_{i}^{(q)}$ and the collective preference degree $\bar{\eta}_{i}$, and then the cardinal consensus index $C C I^{(q)}$ can be calculated. Step 5 is used to find the expert who fails to reach the acceptable consensus level and the feedback strategy that includes identification and direction rules is used to reach the acceptable consensus. Step 6 employs the extended Borda rule to obtain comprehensive utilities after all experts reaching the acceptable consensus, and the optimal alternative is selected based on the comprehensive ranks of alternatives. The procedure of the cardinal consensusbased HFL-DNMA method can be presented in Figure 1 intuitively.

\section{Case study: selecting the optimal geographical location of a large-scale shopping mall}

In this section, a case concerning selecting the optimal geographical location of a shopping mall in the new urbanization is studied. The optimal alternative is selected by the cardinal consensus-based HFL-DNMA method. The advantages of the HFL-DNMA method are showed by comparative analyses with other ranking methods.

\subsection{Case description}

With the booming of China's economy, the number of people who move to cities continues to grow. The process of urbanization has also accelerated dramatically. On March 6, 2019, the press center of the second session of the 13th National People's Congress held a press conference in the Hall of the Median Center. He Lifeng, Director of the National Development and Reform Commission, Ning Jizhe and Lian Weiliang, Vice-Directors of the Commission, were invited to answer questions from Chinese and foreign journalists on issues related to "vigorously promoting high-quality economic development", and they pointed out that the development of new urbanization in China is accelerating. Currently, there are more than 600 cities in China. Among these cities, more than 400 are large and medium-sized cities. Last year, the urbanization rate of the permanent population reached to $59.58 \%$. With each percentage point's increase in the urbanization rate, nearly 14 million people moved from rural areas to urban areas. This not only increases investment in urban public service facilities, but also releases huge consumption demand for food, clothing, housing and transportation. At the same time, during the period of making up for the shortcomings of development, speeding up the transformation of shantytowns and accelerating the transformation of old urban districts, it may release the huge potential consuming market.

It is known that shopping malls are indispensable in a city. The shopping mall improves the living standards of people and driving the economic growth of this area. In the process of new urbanization, how to choose the geographical location of a shopping mall to obtain maximum benefits plays a vital role in the future development. In this section, we use the proposed cardinal consensus-based HFL-DNMA method to solve a case study about selecting the optimal geographical location of a large-scale shopping mall. Suppose that a construction investment agency is preparing to invest in a large-scale shopping mall in the new urbanization and there are five candidate locations which are represented by $A=\left\{a_{1}, a_{2}, a_{3}, a_{4}\right\}$. There are five criteria on the alternatives. $c_{1}$ and $c_{2}$ are benefit criteria. $c_{3}$ and $c_{4}$ are cost criteria. $c_{5}$ is the target criteria. For benefit criteria, they can increase the consumption of shopping malls and its profit income. For cost criteria, they spend on all kinds of expenditure or reduce the overall income. For target criteria, they are neither as low as possible nor as high as possible for the evaluation of a certain alternative, but a value in a range that is most beneficial for the entire alternative. The criteria without intersection among them for evaluating the alternatives are mainly as follows:

- Municipal construction ( $c_{1}$, benefit criterion): the more perfect the municipal construction of the target area is, the more comfortable the shopping environment is and the better the effect of attracting consumers will be. Under normal circumstances, the enterprises around the municipal units are intensive, the mobility of personnel is large, and the potential consumption demand is greatly increased. In addition, municipal units need a lot of office supplies, which also will bring huge benefits to the shopping mall.

- Transportation convenience ( $c_{2}$, benefit criterion): the smoother the traffic condition of the destination is, the more convenient and economical it is for consumers to reach the destination through various means of transport and thus the more shopping groups and repeat customers there will be. The convenience of reaching the mall has become one of the key factors for consumers to be willing to come to spend. It will promote the consumption of surrounding residents and mobile workers.

- Characteristics and scope of business circle $\left(c_{3}\right.$, cost criterion): distribution and marketing strategies of competitors will compete with their own shopping centers. This may increase competition and reduce the profits of the shopping mall. If there are already a large number of shopping malls around the shopping mall location and the supporting facilities are complete, it will undoubtedly affect the sales ability of the shopping mall and reduce its profit. And it will also increase the risk of the shopping mall being closed due to poor competition.

- Degree of population aggregation $\left(c_{4}\right.$, benefit criterion): the more work units there are, the higher the degree of population aggregation will be. Because of the actual situation of China, the purchasing power of the units such as large-scale organs, institutions 
and enterprises, is relatively high, which will become the most valuable potential customers in the market. The selection of shopping mall location needs to consider the degree of population aggregation. Only when the population aggregation is relatively dense, most of the goods sold in the shopping mall need a large supply, which can increase the overall revenue of the shopping mall.

- Ratio of the land investment in total investment $\left(c_{5}\right.$, target criterion): to achieve a relatively suitable investment in fixed assets in a shopping mall, the proportion of land price is also an important factor to be considered in overall investment.

For the above criteria, the criteria $c_{1}, c_{2}, c_{3}, c_{4}$ are qualitative, and the criterion $c_{5}$ is quantitative. The data of $c_{5}$ has been given in the investment planning. The land investment ratio in total investment of $a_{1}, a_{2}, a_{3}$ and $a_{4}$ is $0.11,0.04,0.07$ and 0.09 , respectively. The given target criterion value of $c_{5}$ is 0.06 .

To guarantee the robustness and practicality of the decision-making results, the construction investment agency invites four experts $e_{q}(q=1,2,3,4)$ with weights $w^{(q)}(q=1,2,3,4)$ to carry out the assessments for the alternatives on qualitative criteria and rank the alternatives based on the overall performance of the provided geographical locations. The LTS that the experts used to assess the alternatives under the first three criteria is denoted as: $\left\{s_{-3}=\right.$ very terrible $(V T), s_{-2}=$ terrible $(T), s_{-1}=$ slightly terrible $(S T), s_{0}=$ medium $(M), s_{1}=$ slightly good $(V T), s_{2}=$ good $(D), s_{3}=$ very good $\left.(V D)\right\}$. The LTS that the experts used to assess the alternatives under the criterion $c_{4}$ is denoted by $\left\{s_{-3}=\right.$ very spars $(V S), s_{-2}=$ sparse $(S), s_{-1}=$ slightly spars

$(S S)=e, s_{0}=$ medium $(M), s_{1}=$ slightly dense $(S D), s_{2}=$ dense $(D), s_{3}=$ very dense $\left.(V D)\right\}$. Based on these LTSs, each expert gives flexible linguistic expressions after assessing the alternatives. The linguistic assessments of the alternatives given by the four experts are tabulated in Table 1 .

Next, we use the cardinal consensus-based HFL-DNMA method to solve this MEMCDM problem.

Step 1. By employing the transformation function $E_{G H}$ for qualitative linguistic terms, the decision matrix of each expert is represented as follows:

$D^{(1)}=\left[\begin{array}{ccccc}\left\{s_{1}, s_{2}, s_{3}\right\} & \left\{s_{1}, s_{2}\right\} & \left\{s_{-1}, s_{0}, s_{1}\right\} & \left\{s_{1}, s_{2}\right\} & 0.11 \\ \left\{s_{-1}, s_{0}, s_{1}, s_{2}\right\} & \left\{s_{0}, s_{1}, s_{2}\right\} & \left\{s_{0}\right\} & \left\{s_{-1}, s_{0}, s_{1}\right\} & 0.04 \\ \left\{s_{1}, s_{2}, s_{3}\right\} & \left\{s_{0}, s_{1}, s_{2}\right\} & \left\{s_{0}\right\} & \left\{s_{-1}, s_{0}\right\} & 0.07 \\ \left\{s_{2}\right\} & \left\{s_{1}, s_{2}\right\} & \left\{s_{1}, s_{2}\right\} & \left\{s_{0}, s_{1}\right\} & 0.09\end{array}\right] ;$ $D^{(2)}=\left[\begin{array}{ccccc}\left\{s_{1}, s_{2}\right\} & \left\{s_{0}, s_{1}\right\} & \left\{s_{2}\right\} & \left\{s_{1}, s_{2}\right\} & 0.11 \\ \left\{s_{-1}, s_{0}, s_{1}\right\} & \left\{s_{0}, s_{1}\right\} & \left\{s_{1}, s_{2}\right\} & \left\{s_{-2}, s_{-1}, s_{0}, s_{1}\right\} & 0.04 \\ \left\{s_{-1}, s_{0}\right\} & \left\{s_{-1}, s_{0}\right\} & \left\{s_{1}\right\} & \left\{s_{-2}, s_{-1}, s_{0}\right\} & 0.07 \\ \left\{s_{1}, s_{2}\right\} & \left\{s_{2}\right\} & \left\{s_{2}, s_{3}\right\} & \left\{s_{1}, s_{2}\right\} & 0.09\end{array}\right] ;$

$$
\begin{aligned}
D^{(3)} & =\left[\begin{array}{ccccc}
\left\{s_{-1}, s_{0}, s_{1}, s_{2}\right\} & \left\{s_{2}\right\} & \left\{s_{-1}, s_{0}, s_{1}\right\} & \left\{s_{2}\right\} & 0.11 \\
\left\{s_{0}\right\} & \left\{s_{1}, s_{2}, s_{3}\right\} & \left\{s_{1}, s_{2}, s_{3}\right\} & \left\{s_{-2}, s_{-1}, s_{0}\right\} & 0.04 \\
\left\{s_{2}, s_{3}\right\} & \left\{s_{1}\right\} & \left\{s_{-1}, s_{0}, s_{1}, s_{2}\right\} & \left\{s_{2}\right\} & 0.07 \\
\left\{s_{1}, s_{2}\right\} & \left\{s_{1}, s_{2}\right\} & \left\{s_{2}\right\} & \left\{s_{0}, s_{1}, s_{2}\right\} & 0.09
\end{array}\right] ; \\
D^{(4)} & =\left[\begin{array}{ccccc}
\left\{s_{2}\right\} & \left\{s_{0}, s_{1}\right\} & \left\{s_{0}, s_{1}\right\} & \left\{s_{2}, s_{3}\right\} & 0.11 \\
\left\{s_{-2}, s_{-1}, s_{0}, s_{1}\right\} & \left\{s_{0}, s_{1}, s_{2}, s_{3}\right\} & \left\{s_{2}\right\} & \left\{s_{0}, s_{1}\right\} & 0.04 \\
\left\{s_{0}, s_{1}, s_{2}, s_{3}\right\} & \left\{s_{-1}, s_{0}, s_{1}\right\} & \left\{s_{1}, s_{2}\right\} & \left\{s_{-3}, s_{-2}, s_{-1}, s_{0}\right\} & 0.07 \\
\left\{s_{1}\right\} & \left\{s_{1}, s_{2}\right\} & \left\{s_{1}, s_{2}\right\} & \left\{s_{-2}, s_{-1}, s_{0}\right\} & 0.09
\end{array}\right] .
\end{aligned}
$$

\begin{tabular}{|c|c|c|c|c|}
\hline$e_{1}$ & $c_{1}$ & $c_{2}$ & $c_{3}$ & $c_{4}$ \\
\hline$a_{1}$ & Between SD and VD & Between SD and D & Between ST and SD & Between SD and D \\
\hline$a_{2}$ & Between ST and D & Between $\mathrm{M}$ and $\mathrm{D}$ & $\mathrm{M}$ & Between SS and SD \\
\hline$a_{3}$ & At least SD & Between $\mathrm{M}$ and $\mathrm{D}$ & $\mathrm{D}$ & Between SS and M \\
\hline$a_{4}$ & $\mathrm{D}$ & Between SD and D & Between SD and D & Between $\mathrm{M}$ and SD \\
\hline \multicolumn{5}{|l|}{$e_{2}$} \\
\hline$a_{1}$ & Between SD and D & Between $\mathrm{M}$ and $\mathrm{SD}$ & $\mathrm{D}$ & Between SD and D \\
\hline$a_{2}$ & Between ST and SD & Between $\mathrm{M}$ and SD & Between SD and D & Between $S$ and SD \\
\hline$a_{3}$ & Between ST and M & Between ST and M & SD & Between $S$ and $M$ \\
\hline$a_{4}$ & Between SD and D & $\mathrm{D}$ & Between D and VD & Between SD and D \\
\hline \multicolumn{5}{|l|}{$e_{3}$} \\
\hline$a_{1}$ & Between ST and D & $\mathrm{D}$ & Between ST and SD & $\mathrm{D}$ \\
\hline$a_{2}$ & $\mathrm{M}$ & At least SD & At least SD & Between SS and SD \\
\hline$a_{3}$ & Between SD and D & Between SD and D & $\mathrm{D}$ & Between $\mathrm{S}$ and $\mathrm{M}$ \\
\hline$a_{4}$ & Between D and VD & $\mathrm{SD}$ & Between ST and D & $\mathrm{D}$ \\
\hline \multicolumn{5}{|l|}{$e_{4}$} \\
\hline$a_{1}$ & $\mathrm{D}$ & Between $\mathrm{M}$ and SD & Between $\mathrm{M}$ and SD & Between D and VD \\
\hline$a_{2}$ & Between $\mathrm{T}$ and SD & At least $\mathrm{M}$ & $\mathrm{D}$ & Between $\mathrm{M}$ and SD \\
\hline$a_{3}$ & At least $\mathrm{M}$ & Between ST and SD & Between SD and D & Between VS and M \\
\hline$a_{4}$ & $\mathrm{SD}$ & Between SD and D & Between SD and D & Between $\mathrm{S}$ and $\mathrm{M}$ \\
\hline
\end{tabular}

Table 1. The linguistic assessments of the alternatives given by the four experts 
The HFLEs in these four decision matrices on qualitative criteria are transformed into crisp values by Eq. (1). Thus, we obtain:

$$
\begin{aligned}
X^{(1)} & =\left[\begin{array}{lllll}
0.633 & 0.675 & 0.380 & 0.675 & 0.11 \\
0.344 & 0.507 & 0.500 & 0.380 & 0.04 \\
0.633 & 0.507 & 0.833 & 0.375 & 0.07 \\
0.833 & 0.675 & 0.833 & 0.525 & 0.09
\end{array}\right] ; \\
X^{(2)} & =\left[\begin{array}{lllll}
0.675 & 0.525 & 0.833 & 0.675 & 0.11 \\
0.380 & 0.525 & 0.675 & 0.246 & 0.04 \\
0.525 & 0.375 & 0.675 & 0.375 & 0.04 \\
0.675 & 0.833 & 0.825 & 0.675 & 0.09
\end{array}\right] ; \\
X^{(3)} & =\left[\begin{array}{lllll}
0.344 & 0.833 & 0.380 & 0.833 & 0.11 \\
0.500 & 0.633 & 0.633 & 0.380 & 0.04 \\
0.825 & 0.667 & 0.344 & 0.833 & 0.07 \\
0.675 & 0.675 & 0.833 & 0.507 & 0.09
\end{array}\right] ; \\
X^{(4)}= & {\left[\begin{array}{lllll}
0.833 & 0.525 & 0.525 & 0.697 & 0.11 \\
0.246 & 0.443 & 0.833 & 0.525 & 0.04 \\
0.443 & 0.380 & 0.675 & 0.148 & 0.07 \\
0.667 & 0.675 & 0.675 & 0.253 & 0.09
\end{array}\right] . }
\end{aligned}
$$

Based on the above decision matrices, by Eq. (6), we calculate the target-based linear normalized values. The calculated results are illustrated in Table 2.

Table 2. The target-based linear normalized values of the four experts

\begin{tabular}{|c|c|c|c|c|c|}
\hline$e_{1}$ & $c_{1}$ & $c_{2}$ & $c_{3}$ & $c_{4}$ & $e_{1}$ \\
\hline$a_{1}$ & 0.591 & 1.000 & 1.000 & 0.591 & 0.000 \\
\hline$a_{2}$ & 0.000 & 0.000 & 0.735 & 0.000 & 0.600 \\
\hline$a_{3}$ & 0.591 & 0.000 & 0.000 & 0.591 & 0.800 \\
\hline$a_{4}$ & 1.000 & 1.000 & 0.349 & 1.000 & 0.400 \\
\hline$e_{2}$ & & & & & \\
\hline$a_{1}$ & 1.000 & 0.328 & 0.000 & 1.000 & 0.000 \\
\hline$a_{2}$ & 0.017 & 0.328 & 0.952 & 0.000 & 0.600 \\
\hline$a_{3}$ & 0.500 & 0.000 & 0.952 & 0.301 & 0.800 \\
\hline$a_{4}$ & 1.000 & 1.000 & 0.048 & 1.000 & 0.400 \\
\hline$e_{3}$ & & & & & \\
\hline$a_{1}$ & 0.000 & 1.000 & 0.926 & 1.000 & 0.000 \\
\hline$a_{2}$ & 0.324 & 0.000 & 0.409 & 0.000 & 0.600 \\
\hline$a_{3}$ & 1.000 & 0.170 & 1.000 & 1.000 & 0.800 \\
\hline$a_{4}$ & 0.688 & 0.210 & 0.000 & 0.280 & 0.400 \\
\hline$e_{4}$ & & & & & \\
\hline$a_{1}$ & 1.000 & 0.492 & 1.000 & 1.000 & 0.000 \\
\hline$a_{2}$ & 0.000 & 0.214 & 0.000 & 0.687 & 0.600 \\
\hline$a_{3}$ & 0.336 & 0.000 & 0.513 & 0.000 & 0.800 \\
\hline$a_{4}$ & 0.717 & 1.000 & 0.513 & 0.191 & 0.400 \\
\hline & & & & & \\
\hline & & & & & \\
\hline
\end{tabular}

By Eq. (7), we calculate the target-based vector normalized values. The results are presented in Table 3.
Table 3. The target-based vector normalized values of the four experts

\begin{tabular}{|l|c|c|c|c|c|}
\hline$e_{1}$ & $c_{1}$ & $c_{2}$ & $c_{3}$ & $c_{4}$ & $e_{1}$ \\
\hline$a_{1}$ & 0.868 & 1.000 & 1.000 & 1.000 & 0.713 \\
\hline$a_{2}$ & 0.678 & 0.878 & 0.908 & 0.757 & 0.885 \\
\hline$a_{3}$ & 0.868 & 0.878 & 0.651 & 0.753 & 0.943 \\
\hline$a_{4}$ & 1.000 & 1.000 & 0.773 & 0.876 & 0.828 \\
\hline$e_{2}$ & & & & & \\
\hline$a_{1}$ & 1.000 & 0.786 & 0.900 & 1.000 & 0.713 \\
\hline$a_{2}$ & 0.779 & 0.786 & 0.995 & 0.657 & 0.885 \\
\hline$a_{3}$ & 0.888 & 0.682 & 0.995 & 0.760 & 0.943 \\
\hline$a_{4}$ & 1.000 & 1.000 & 0.904 & 1.000 & 0.828 \\
\hline$e_{3}$ & & & & & \\
\hline$a_{1}$ & 0.675 & 1.000 & 0.970 & 1.000 & 0.713 \\
\hline$a_{2}$ & 0.780 & 0.878 & 0.762 & 0.713 & 0.885 \\
\hline$a_{3}$ & 1.000 & 0.899 & 1.000 & 1.000 & 0.943 \\
\hline$a_{4}$ & 0.899 & 0.904 & 0.597 & 0.793 & 0.828 \\
\hline$e_{4}$ & & & & & \\
\hline$a_{1}$ & 1.000 & 0.879 & 1.000 & 1.000 & 0.713 \\
\hline$a_{2}$ & 0.594 & 0.812 & 0.790 & 0.851 & 0.885 \\
\hline$a_{3}$ & 0.730 & 0.761 & 0.898 & 0.525 & 0.943 \\
\hline$a_{4}$ & 0.885 & 1.000 & 0.898 & 0.615 & 0.828 \\
\hline$A_{s}$ & & & & & \\
\hline
\end{tabular}

Assume that the construction investment agency determines the weights of the five criteria as: $\omega_{1}=0.28, \omega_{2}=0.22$, $\omega_{3}=0.18, \omega_{4}=0.20$ and $\omega_{5}=0.12$. Based on the results of two normalization methods, by Eqs (8)-(10), the values of CCM, UCM and ICM are calculated for each expert, respectively. Meanwhile, the alternatives are ranked under each subordinate aggregation model. According to the three types of calculated subordinate utility values and the corresponding ranks of alternatives, by Eq. (11) and Eq. (5), the integrated scores and ranks of alternatives for each expert can be obtained. The above calculated results of each expert are presented in Tables 4-7.

Since different experts have different knowledge background and experience, the integrated scores and ranks of different experts have differences. Next, the consensus degree of each expert needs to be taken into account. Based on Eqs (12)-(15), the calculated results for the cardinal consensus reaching process can be expressed in Table 8 .

We can obtain $C C I^{(1)}=0.922, \quad C C I^{(2)}=0.922$, $C C I^{(3)}=0.836$ and $C C I^{(4)}=0.922$. Since the geographical location of a larger-scale shopping mall is vital for a construction investment agency and the number of experts is small, the consensus threshold $\lambda$ is set as 0.9 . According to Eq. (16), the expert $e_{3}$ does not reach the predefined consensus threshold $\gamma=0.9$. Thus, the linguistic assessments of expert $e_{3}$ for a certain alternative should be modified. By Eq. (17), the concrete alternative that needs to be modified by expert $e_{3}$ can be identified, that is, the linguistic preference assessments of expert $e_{3}$ on alternative $a_{3}$ should be decreased on different criteria. Giving feedback advices to expert $e_{3}$, new linguistic assessments can be obtained under multiple criteria. 
Table 4 . The calculated results derived by the HFL-DNMA method for the expert $e_{1}$

\begin{tabular}{|c|c|c|c|c|c|c|c|c|c|c|c|}
\hline & \multicolumn{3}{|c|}{ CCM } & \multicolumn{3}{|c|}{ UCM } & \multicolumn{3}{|c|}{ ICM } & \multirow{2}{*}{$\begin{array}{l}\text { Integrated } \\
\text { score }\end{array}$} & \multirow{2}{*}{ Ranks } \\
\hline & $u_{1}^{(1)}\left(a_{i}\right)$ & $r_{1}^{(1)}\left(a_{i}\right)$ & $u_{1}^{(1) N}\left(a_{i}\right)$ & $u_{2}^{(1)}\left(a_{i}\right)$ & $r_{2}^{(1)}\left(a_{i}\right)$ & $u_{2}^{(1) N}\left(a_{i}\right)$ & $u_{3}^{(1)}\left(a_{i}\right)$ & $r_{3}^{(1)}\left(a_{i}\right)$ & $u_{3}^{(1) N}\left(a_{i}\right)$ & & \\
\hline$a_{1}$ & 0.765 & 1 & 0.635 & 0.120 & 2 & 0.100 & 0.923 & 4 & 0.766 & 0.919 & 2 \\
\hline$a_{2}$ & 0.208 & 4 & 0.238 & 0.280 & 4 & 0.321 & 0.798 & 2 & 0.916 & 0.502 & 4 \\
\hline$a_{3}$ & 0.261 & 3 & 0.297 & 0.220 & 3 & 0.250 & 0.811 & 1 & 0.922 & 0.687 & 3 \\
\hline$a_{4}$ & 0.711 & 2 & 0.613 & 0.117 & 1 & 0.101 & 0.909 & 3 & 0.784 & 0.954 & 1 \\
\hline
\end{tabular}

Table 5. The calculated results derived by the HFL-DNMA method for the expert $e_{2}$

\begin{tabular}{|c|c|c|c|c|c|c|c|c|c|c|c|}
\hline & \multicolumn{3}{|c|}{ CCM } & \multicolumn{3}{c|}{ UCM } & \multicolumn{3}{c|}{ ICM } & Integrated \\
\cline { 2 - 13 } & $u_{1}^{(2)}\left(a_{i}\right)$ & $r_{1}^{(2)}\left(a_{i}\right)$ & $u_{1}^{(2) N}\left(a_{i}\right)$ & $u_{2}^{(2)}\left(a_{i}\right)$ & $r_{2}^{(2)}\left(a_{i}\right)$ & $u_{2}^{(2) N}\left(a_{i}\right)$ & $u_{3}^{(2)}\left(a_{i}\right)$ & $r_{3}^{(2)}\left(a_{i}\right)$ & $u_{3}^{(2) N}\left(a_{i}\right)$ & $\begin{array}{c}\text { Ranks } \\
\text { score }\end{array}$ \\
\hline$a_{1}$ & 0.552 & 2 & 0.503 & 0.180 & 2 & 0.164 & 0.931 & 3 & 0.848 & 0.848 & 2 \\
\hline$a_{2}$ & 0.320 & 4 & 0.327 & 0.275 & 4 & 0.281 & 0.884 & 1 & 0.902 & 0.594 & 4 \\
\hline$a_{3}$ & 0.467 & 3 & 0.449 & 0.280 & 3 & 0.269 & 0.888 & 2 & 0.852 & 0.702 & 3 \\
\hline$a_{4}$ & 0.757 & 1 & 0.605 & 0.171 & 1 & 0.137 & 0.982 & 4 & 0.785 & 0.952 & 1 \\
\hline
\end{tabular}

Table 6 . The calculated results derived by the HFL-DNMA method for the expert $e_{3}$

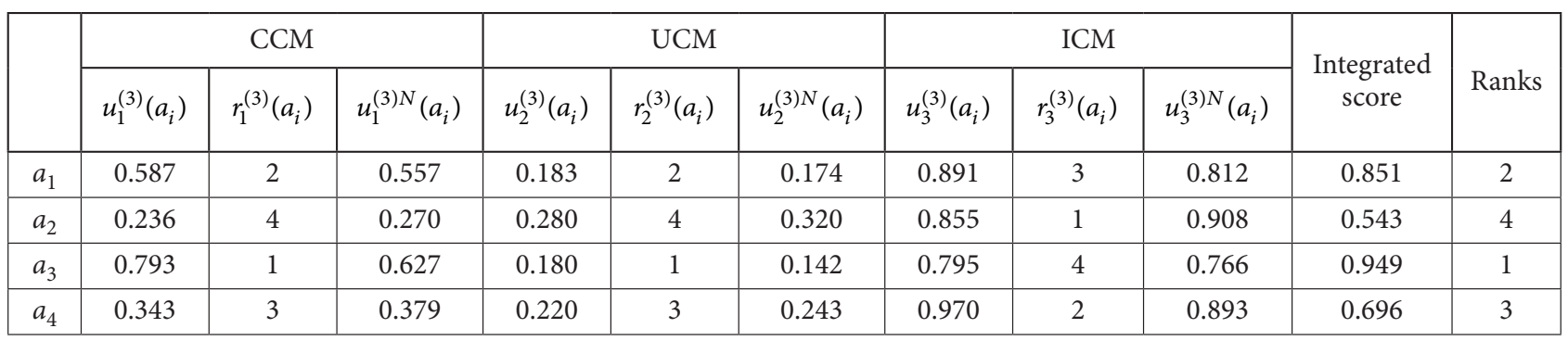

Table 7. The calculated results derived by the HFL-DNMA method for the expert $e_{4}$

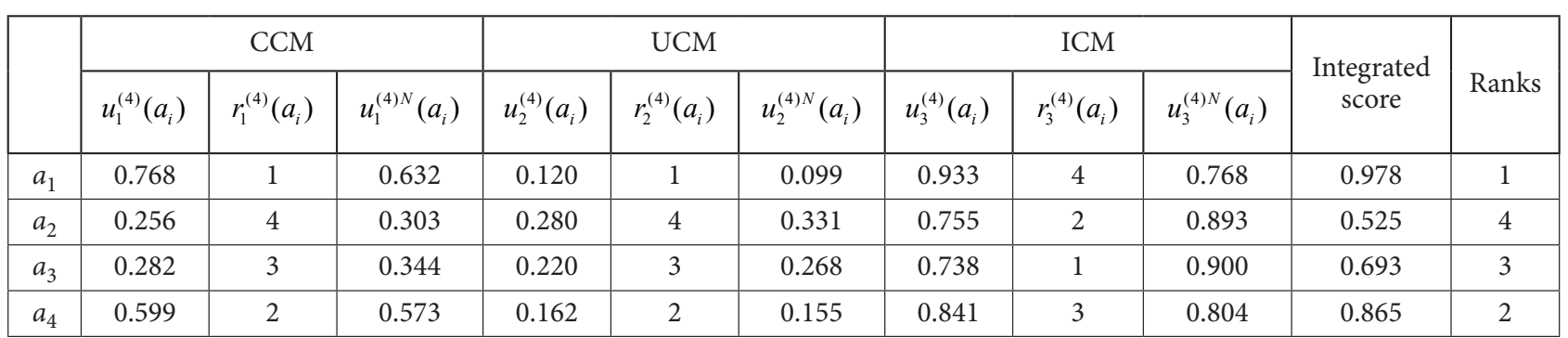

Table 8. The calculated results of the cardinal consensus reaching process

\begin{tabular}{|c|c|c|c|c|c|}
\hline$\eta_{i}^{(q)}$ & $a_{1}$ & $a_{2}$ & $a_{3}$ & $a_{4}$ & $C C I^{(q)}$ \\
\hline$e_{1}$ & 0.333 & 0 & 0.167 & 0.5 & 0.922 \\
\hline$e_{2}$ & 0.333 & 0 & 0.167 & 0.5 & 0.922 \\
\hline$e_{3}$ & 0.333 & 0 & 0.5 & 0.167 & 0.836 \\
\hline$e_{4}$ & 0.5 & 0 & 0.167 & 0.333 & 0.922 \\
\hline$\overline{\eta_{i}}$ & 0.375 & 0 & 0.25 & 0.375 & - \\
\hline
\end{tabular}

Here, to save space, the first modified decision-making matrix for expert $e_{3}$ is directly presented as:

$$
D^{(3)^{1}}=\left[\begin{array}{cccc}
\left\{s_{-1}, s_{0}, s_{1}, s_{2}\right\} & \left\{s_{2}\right\} & \left\{s_{-1}, s_{0}, s_{1}\right\} & \left\{s_{2}\right\} \\
\left\{s_{0}\right\} & \left\{s_{1}, s_{2}, s_{3}\right\} & \left\{s_{1}, s_{2}, s_{3}\right\} & \left\{s_{-2}, s_{-1}, s_{0}\right\} \\
\left\{s_{1}, s_{2}\right\} & \left\{s_{-1}, s_{0}, s_{1}\right\} & \left\{s_{1}, s_{2}\right\} & \left\{s_{2}\right\} \\
\left\{s_{1}, s_{2}\right\} & \left\{s_{1}, s_{2}\right\} & \left\{s_{2}\right\} & \left\{s_{0}, s_{1}, s_{2}\right\}
\end{array}\right] .
$$

After the first feedback round of expert $e_{3}$, by Eqs (12)(15), the calculated results of $C C I^{(q)}$ are $C C I^{(1)}=0.922$, $C C I^{(2)}=0.922, C C I^{(3)}=0.872$ and $C C I^{(4)}=0.949$. By Eqs (16) and (17), the linguistic preference assessments of 
expert $e_{3}$ on alternative $a_{4}$ should be increased. The second modified decision matrix for expert $e_{3}$ is shown as:

$D^{(3)^{2}}=\left[\begin{array}{cccc}\left\{s_{-1}, s_{0}, s_{1}, s_{2}\right\} & \left\{s_{2}\right\} & \left\{s_{-1}, s_{0}, s_{1}\right\} & \left\{s_{2}\right\} \\ \left\{s_{0}\right\} & \left\{s_{1}, s_{2}, s_{3}\right\} & \left\{s_{1}, s_{2}, s_{3}\right\} & \left\{s_{-2}, s_{-1}, s_{0}\right\} \\ \left\{s_{1}, s_{2}\right\} & \left\{s_{-1}, s_{0}, s_{1}\right\} & \left\{s_{1}, s_{2}\right\} & \left\{s_{2}\right\} \\ \left\{s_{1}, s_{2}\right\} & \left\{s_{1}, s_{2}\right\} & \left\{s_{0}, s_{1}\right\} & \left\{s_{0}, s_{1}\right\}\end{array}\right]$.

After the second feedback round for expert $e_{3}$, the calculated results of $C C I^{(q)}$ are $C C I^{(1)}=0.941$, $C C I^{(2)}=0.941, C C I^{(3)}=0.941$ and $C C I^{(4)}=0.941$. It is obvious that all $C C I^{(q)}$ of the experts are larger than the predefined consensus threshold $\lambda=0.9$. In other words, all experts reach the acceptable consensus degree.

According to the proposed extended Borda rule, we can aggregate the integrated scores and subordinate ranks of alternatives obtained by each expert to obtain a comprehensive rank. Here, we need to use the final modified results of expert $e_{3}$, and the final results derived by the HFL-DNMA method based on the modified linguistic assessments are shown in Table 9.

Based on Tables 4, 5, 7 and 9, we can obtain the integrated scores $S_{i}$ and subordinate ranks $R$ of four experts. Without lose of generality, the weight of each expert is set as $w^{(q)}=0.25(q=1,2,3,4)$. By Eqs (18) and (19), we can obtain $C U\left(a_{1}\right)=1.303, C U\left(a_{2}\right)=0.704, C U\left(a_{3}\right)=0.875$ and $C U\left(a_{4}\right)=1.287$. Based on the comprehensive utility value of each alternative, the final ranking of the four alternatives is obtained as $R\left(a_{1}\right) \succ R\left(a_{4}\right) \succ R\left(a_{3}\right) \succ R\left(a_{2}\right)$, where " $\succ$ " denotes "superior to". Thus, for this construction investment agency, the alternative $a_{1}$ is the optimal geographical location to construct a larger-scale shopping mall.

\subsection{Comparative analyses with other ranking methods}

To illustrate the feasibility and advantages of the cardinal consensus-based HFL-DNMA method, we compare the proposed method with other ranking methods, including the HFL-TOPSIS (Beg \& Rashid, 2013) and HFL-VIKOR (Liao et al., 2015), which have been widely applied to rank alternatives in MCDM problems.

To show the differences between different methods, the weights of criteria and experts keep the same as given in the HFL-DNMA method. Firstly, we integrate the decision matrices, $X^{(1)}, X^{(2)}, X^{(3)}$ and $X^{(4)}$, of four experts to a collective one, which is presented as follows:

$$
X=\left[\begin{array}{lllll}
0.621 & 0.640 & 0.530 & 0.720 & 0.110 \\
0.368 & 0.527 & 0.660 & 0.383 & 0.040 \\
0.607 & 0.482 & 0.632 & 0.433 & 0.070 \\
0.713 & 0.715 & 0.752 & 0.490 & 0.090
\end{array}\right]
$$

Then, we use two different ranking methods to solve the above case.

(1) Solving the case by the HFL-VIKOR method

Here, the HFL-VIKOR method (Liao et al., 2015) is extended to handle both qualitative and quantitative information. For the collective matrix $X$, the target-based linear normalized values can be obtained by Eq. (6), which are presented in Table 10. The compromise value of each alternative can be calculated by $C V_{i}=\theta\left(G U_{i}-\right.$ $\left.G U^{-}\right) /\left(G U^{+}-G U^{-}\right)+(1-0)\left(I R^{+}-I R_{i}\right) /\left(I R^{+}-I R^{-}\right)$. Here, $G U_{i}=\sum_{j=1}^{n} \omega_{j} \kappa_{i j}^{1}$ denotes the group utility of alternative $a_{1}$, and $I R_{i}=\max _{j}\left(\omega_{j}\left(1-\kappa_{i j}^{1}\right)\right)$ denotes the individual regret value. Besides, $G U^{+}=\max _{i} G U_{i}, G U^{-}=\min _{i} G U_{i}$, $I R^{+}=\max _{i} I R_{i}$ and $I R^{-}=\min _{i} I R_{i} . \theta$ denotes the importance on ${ }^{i} G U_{i}$ or $I R_{i}$.

If $\theta=0.3$, we can calculate that $C V_{1}=1, C V_{2}=0$, $C V_{3}=0,383, V_{4}=0.655$, and thus $R_{\theta=0.3}=\{1,4,3,2\}$. If $\theta=0.8$, we can calculate that $C V_{1}=0.999, C V_{2}=0$, $C V_{3}=0.409, V_{4}=0.744$, and thus $R_{\theta=0.8}=\{1,4,3,2\}$.

Table 9. The results calculated by the HFL-DNMA method based on the modified linguistic assessments of $e_{3}$

\begin{tabular}{|c|c|c|c|c|c|c|c|c|c|c|c|}
\hline & \multicolumn{3}{|c|}{$\mathrm{CCM}$} & \multicolumn{3}{|c|}{ UCM } & \multicolumn{3}{|c|}{ ICM } & \multirow{2}{*}{$\begin{array}{l}\text { Integrated } \\
\text { score }\end{array}$} & \multirow{2}{*}{ Ranks } \\
\hline & $u_{1}^{(3)}\left(a_{i}\right)$ & $r_{1}^{(3)}\left(a_{i}\right)$ & $u_{1}^{(3) N}\left(a_{i}\right)$ & $u_{2}^{(3)}\left(a_{i}\right)$ & $r_{2}^{(3)}\left(a_{i}\right)$ & $u_{2}^{(3) N}\left(a_{i}\right)$ & $u_{3}^{(3)}\left(a_{i}\right)$ & $r_{3}^{(3)}\left(a_{i}\right)$ & $u_{3}^{(3) N}\left(a_{i}\right)$ & & \\
\hline$a_{1}$ & 0.587 & 2 & 0.560 & 0.183 & 2 & 0.175 & 0.849 & 3 & 0.810 & 0.910 & 1 \\
\hline$a_{2}$ & 0.236 & 4 & 0.272 & 0.280 & 4 & 0.322 & 0.789 & 1 & 0.907 & 0.487 & 4 \\
\hline$a_{3}$ & 0.269 & 1 & 0.298 & 0.180 & 1 & 0.200 & 0.840 & 4 & 0.933 & 0.771 & 3 \\
\hline$a_{4}$ & 0.464 & 3 & 0.464 & 0.220 & 3 & 0.220 & 0.859 & 2 & 0.858 & 0.797 & 2 \\
\hline
\end{tabular}

Table 10. The target-based linear normalized values

\begin{tabular}{|c|c|c|c|c|c|}
\hline & $c_{1}$ & $c_{2}$ & $c_{3}$ & $c_{4}$ & $c_{5}$ \\
\hline$a_{1}$ & 0.733 & 0.678 & 1.000 & 1.000 & 0.000 \\
\hline$a_{2}$ & 0.000 & 0.193 & 0.414 & 0.000 & 0.600 \\
\hline$a_{3}$ & 0.693 & 0.000 & 0.541 & 0.148 & 0.800 \\
\hline$a_{4}$ & 1.000 & 1.000 & 0.000 & 0.318 & 0.400 \\
\hline
\end{tabular}


(2) Solving the case by the HFL-TOPSIS method

To solve the case with both quantitative and qualitative criteria, we extend the HFL-TOPSIS (Beg \& Rashid, 2013) by combining it with both target-based linear normalization and vector normalization. The target-based linear normalized values of the collective matrix $X$ is denoted in Table 10. The target-based vector normalized values on the collective matrix $X$ is calculated by Eq. (2), which are illustrated in Table 11. Based on Table 10 and Table 11, the distance of each alternative to the ideal solution can be calculated by $D_{i}^{+}=\sqrt{\sum_{j=1}^{n} \omega_{j}\left(\kappa_{i j}^{1}-\kappa_{j}^{+}\right)^{2}}$, and the distance of each alternative to the negative-ideal solution can be calculated by $D_{i}^{-}=\sqrt{\sum_{j=1}^{n} \omega_{j}\left(\kappa_{i j}^{1}-\kappa_{j}^{-}\right)^{2}}$, in which $\kappa_{j}^{+}=\max _{i} \kappa_{i j}^{1}, \kappa_{j}^{-}=\min _{i} \kappa_{i j}^{1} . R C_{i}=D_{i}^{-} /\left(D_{i}^{+}+D_{i}^{-}\right)$ denotes the relative closeness to the ideal solution. The ranks of alternatives are obtained in descending order of $R C_{i}(i=1,2,3,4)$.

For target-based linear normalized values, we can obtain $R C_{1}=0.697, R C_{2}=0.257, R C_{3}=0.441, R C_{4}=0.576$. Thus, the rank set of the alternatives is $R_{1}=\{1,4,3,2\}$. For target-based vector normalized values, we can obtain $R C_{1}=0.659, R C_{2}=0.258, R C_{3}=0.481, R C_{4}=0.592$. Thus, the rank set of the alternatives is $R_{1}=\{1,4,3,2\}$.

Based on the final results of all the ranking methods, we can obtain that alternative $a_{1}$ is the optimal choice, and alternative $a_{2}$ is the worst option for the location of shopping mall.

For the extended HFL-VIKOR method, if the number of alternatives is large, the final ranking results may be influenced by the parameter $\theta$. The value of $\theta$ is also difficult to be appropriately determined, which may bias the final result. Besides, for the HFL-TOPSIS method, the ranking results are the same with respect to two different normalization operators. However, the HFL-TOPSIS does not consider the consensus of experts, and the normalized numerical values might exist bias. For the above two methods, since they directly aggregate linguistic assessments of four experts into a collective decision-making matrix, it is easy to loss original information of experts. Whether each expert has reached a consensus is not considered. The final decision-making results may appear some differences. For the proposed method, we consider three subordinate ranks and integrated scores of alternatives. In addition, we also take the consensus degrees of experts into account, which contributes to modifying the linguistic assessments of experts to reach a high-level

Table 11. The target-based vector normalized values

\begin{tabular}{|c|c|c|c|c|c|}
\hline & $c_{1}$ & $c_{2}$ & $c_{3}$ & $c_{4}$ & $c_{5}$ \\
\hline$a_{1}$ & 0.933 & 0.946 & 1.000 & 1.000 & 0.713 \\
\hline$a_{2}$ & 0.750 & 0.865 & 0.907 & 0.734 & 0.885 \\
\hline$a_{3}$ & 0.923 & 0.833 & 0.927 & 0.774 & 0.943 \\
\hline$a_{4}$ & 1.000 & 1.000 & 0.842 & 0.819 & 0.828 \\
\hline
\end{tabular}

consensus. Then, we employ the extended Borda rule to aggregate subordinate ranks and integrated scores to derive a comprehensive ranking of all alternatives, which can reduce the loss of decision-making information for experts and make the ultimate result objective and reliable. According to above analysis, the cardinal consensusbased HFL-DNMA method is more comprehensive than the other ranking methods.

\section{Conclusions}

In the process of MEMCDM, the assessments of alternatives are not only in quantitative forms, but also in qualitative expressions. In addition, the criteria for evaluating the alternatives may have different types, such as the benefit, cost and target criteria. To solve these problems, in this study, we proposed a comprehensive MEMCDM method named the cardinal consensus-based HFL-DNMA method. The advantages of the proposed method can be shown in the following aspects:

1. The HFL-DNMA method provided a new way to deal with MEMCDM problems to obtain the optimal alternative in the hesitant fuzzy linguistic environment.

2. Two normalized methods and three subordinate aggregation models were combined to obtain the comprehensive values of alternatives, which avoided the difficulty of transforming different dimensions under different criteria and the loss of evaluation information.

3. The preference degrees of experts for alternatives were obtained by the original ranks of alternatives. Then, the cardinal consensus process was introduced to help experts reach a high-level consensus.

4. The final comprehensive scores of alternatives were derived by the extended Borda rule to integrate the scores and subordinate ranks of alternatives, which made the result reliable and objective.

In the future, we will consider the case in which the criteria have an impact on each other under the HFL-DNMA framework. The weight of experts will be considered objectively by appropriate reasoning and calculation. We will also extend the DNMA based on intuitionistic fuzzy sets, interval-valued intuitionistic fuzzy sets and Pythagorean fuzzy sets. In addition, it is also meaningful to apply the cardinal consensus-based HFL-DNMA method to other fields to show its feasibility and practicability.

\section{Funding}

This work was supported by the National Natural Science Foundation of China under Grant No. 71771156 and Grant No. 71971145.

\section{Author contributions}

Song Nie and Huchang Liao conceived the study and were responsible for the design and development of the data analysis. Huchang Liao, Xingli Wu and Ming Tang were 
responsible for data collection and analysis. Abdullah AlBarakati were responsible for data interpretation. Song Nie and Huchang Liao wrote the first draft of the article.

\section{Disclosure statement}

The authors have no competing financial, professional, or personal interests from other parties that are related to this paper.

\section{References}

Beg, I., \& Rashid, T. (2013). TOPSIS for hesitant fuzzy linguistic term sets. International Journal of Intelligent Systems, 28(12), 1162-1171. https://doi.org/10.1002/int.21623

Ben-Arieh, D., \& Chen, Z. F. (2006). Linguistic-labels aggregation and consensus measure for autocratic decision-making using group recommendations. IEEE Transactions on Systems, Man, and Cybernetics - Part A: Systems and Humans, 36(3), 558-568. https://doi.org/10.1109/TSMCA.2005.853488

Can, G. F., \& Delice, E. K. (2018). A task-based fuzzy integrated MCDM approach for shopping mall selection considering universal design criteria. Soft Computing, 22(22), 7377-7397. https://doi.org/10.1007/s00500-018-3074-4

Canas, S. R. D., Ferreira, F. A. F., \& Meidutè-Kavaliauskienė, I. (2015). Setting rents in residential real estate: a methodological proposal using multiple criteria decision analysis. International Journal of Strategic Property Management, 19(4), 368380. https://doi.org/10.3846/1648715X.2015.1093562

Cheng, E. W. L., Li, H., \& Yu, L. (2005). The analytic network process (ANP) approach to location selection: a shopping mall illustration. Construction Innovation, 5(2), 83-97. https://doi.org/10.1108/14714170510815195

Cheng, E. W. L., Li, H., \& Yu, L. (2007). A GIS approach to shopping mall location selection. Building and Environment, 42(2), 884-892. https://doi.org/10.1016/j.buildenv.2005.10.010

Dong, Y. C., Zhang, G. Q., Hong, W. C., \& Xu, Y. F. (2010). Consensus models for AHP group decision making under row geometric mean prioritization method. Decision Support Systems, 49(3), 281-289. https://doi.org/10.1016/j.dss.2010.03.003

Elevli, B. (2014). Logistics freight center locations decision by using Fuzzy-PROMETHEE. Transport, 29(4), 412-418. https://doi.org/10.3846/16484142.2014.983966

ElSamen, A. A. A., \& Hiyasat R. I. (2017). Beyond the random location of shopping malls: a GIS perspective in Amman, Jordan. Journal of Retailing and Consumer Services, 34, 30-37. https://doi.org/10.1016/j.jretconser.2016.09.006

Gundogdu, C. E. (2013). Determination of the most suitable sites for shopping centers in geographical regions with GIS. Research in Logistics \& Production, 3(2), 109-122.

Herrera, F., Herrera-Viedma, E., \& Verdegay, J. L. (1996). Direct approach processes in group decision making using linguistic OWA operators. Fuzzy Sets and Systems, 79, 175-190. https://doi.org/10.1016/0165-0114(95)00162-X

Herrera-Viedma, E., Martínez, L., Mata, F., \& Chiclana, F. (2005). A consensus support systems model for group decision making problems with multigranular linguistic preference relations. IEEE Transactions on Fuzzy Systems, 13(5), 644-658. https://doi.org/10.1109/TFUZZ.2005.856561

Kahraman, C., Engin, O., Kabak, O., \& Kaya, I. (2009). Information systems outsourcing decisions using a GDM approach. Engineering Applications of Artificial Intelligence, 22(6), 832841. https://doi.org/10.1016/j.engappai.2008.10.009
Lee, H. S. (2002). Optimal consensus of fuzzy opinions under group decision making environment. Fuzzy Sets and Systems, 132(3), 303-315. https://doi.org/10.1016/S0165-0114(02)00056-8

Li, C. C., Dong, Y. C., Herrera, F., \& Herrera-Viedma, E. (2017). Personalized individual semantics in computing with words for supporting linguistic group decision making. An application on consensus reaching. Information Fusion, 33, 29-40. https://doi.org/10.1016/j.inffus.2016.04.005

Liao, H. C., \& Wu, X. L. (2019). DNMA: a double normalizationbased multiple aggregation method for multi-expert multicriteria decision making. Omega (in press). https://doi.org/10.1016/j.omega.2019.04.001

Liao, H. C., Qin, R., Gao, C. Y., Wu, X. L., Hafezalkotob, A., \& Herrera, F. (2019). Score-HeDLiSF: a score function of hesitant fuzzy linguistic term set based on hesitant degrees and linguistic scale functions: an application to unbalanced hesitant fuzzy linguistic MULTIMOORA. Information Fusion, 48, 39-54. https://doi.org/10.1016/j.inffus.2018.08.006

Liao, H. C., Wu, X. L., Liang, X. D., Xu, J. P., \& Herrera, F. (2018). A new hesitant fuzzy linguistic ORESTE method for hybrid multi-criteria decision making. IEEE Transactions on Fuzzy Systems, 26(6), 3793-3807.

https://doi.org/10.1109/TFUZZ.2018.2849368

Liao, H. C., Wu, X. L., Mi, X. M., \& Herrera, F. (2019). An integrated method for cognitive complex multiple experts multiple criteria decision making based on ELECTRE III with weighted Borda rule. Omega (in press).

https://doi.org/10.1016/j.omega.2019.03.010

Liao, H. C., Xu, Z. S., \& Zeng, X. J. (2015). Hesitant fuzzy linguistic VIKOR method and its application in qualitative multiple criteria decision making. IEEE Transactions on Fuzzy Systems, 23(5), 1343-1355.

https://doi.org/10.1109/TFUZZ.2014.2360556

Liao, H. C., Xu, Z. S., Herrera-Viedma, E., \& Herrera, F. (2018). Hesitant fuzzy linguistic term set and its application in decision making: a state-of-the-art survey. International Journal of Fuzzy Systems, 20(12), 1-27. https://doi.org/10.1007/s40815-017-0432-9

Liao, H. C., Xu, Z. S., Zeng, X. J., \& Merigó, J. M. (2015). Qualitative decision making with correlation coefficients of hesitant fuzzy linguistic term sets. Knowledge-Based Systems, 76, 127138. https://doi.org/10.1016/j.knosys.2014.12.009

Liu, Y., Liang, C., Chiclana, F., \& Wu, J. (2017). A trust induced recommendation mechanism for reaching consensus in group decision making. Knowledge-Based Systems, 119, 221231. https://doi.org/10.1016/j.knosys.2016.12.014

Parreiras, R. O., Ekel, P. Y., Martini, J. S. C., \& Palhares, R. M. (2010). A flexible consensus scheme for multicriteria group decision making under linguistic assessments. Information Sciences, 180(7), 1075-1089.

https://doi.org/10.1016/j.ins.2009.11.046

Perez, I. J., Cabrerizo, F. J., Alonso, S., \& Herrera-Viedma, E. (2014). A new consensus model for group decision making problems with non-homogeneous experts. IEEE Transactions on Systems, Man, and Cybernetics: Systems, 44(4), 494-498. https://doi.org/10.1109/TSMC.2013.2259155

Rodríguez, R. M., Martínez, L., \& Herrera, F. (2012). Hesitant fuzzy linguistic term sets for decision making. IEEE Transactions on Fuzzy Systems, 20(1), 109-119. https://doi.org/10.1109/TFUZZ.2011.2170076

Ross, A., \& Jayaraman, V. (2008). An evaluation of new heuristics for the location of cross-docks distribution centers in supply chain network design. Computers \& Industrial Engineering, 55(1), 64-79. https://doi.org/10.1016/j.cie.2007.12.001 
Song, G. F., Yuan, Y. B., \& Zhang, M. Y. (2008). Shopping mall location based on AHP and multi-level fuzzy comprehensive evaluation. Construction Management Modernization, 6, 13-16.

Torra, V. (2010). Hesitant fuzzy sets. International Journal of Intelligent Systems, 25(6), 529-539. https://doi.org/10.1002/int.20418

Wibowo, S., \& Deng, H. (2013). Consensus-based decision support for multicriteria group decision making. Computers \& Industrial Engineering, 66(4), 625-633. https://doi.org/10.1016/j.cie.2013.09.015

Wu, J., Dai, L. F., Chiclana, F., Fujita, H., \& Herrera-Viedma, E. (2018). A minimum adjustment cost feedback mechanism based consensus model for group decision making under social network with distributed linguistic trust. Information Fusion, 41, 232-242. https://doi.org/10.1016/j.inffus.2017.09.012

Wu, Z. B., \& Xu, J. P. (2018). A consensus model for large-scale group decision making with hesitant fuzzy information and changeable clusters. Information Fusion, 41, 217-231.

https://doi.org/10.1016/j.inffus.2017.09.011
Xu, X. H., Zhong, X. Y., Chen, X. H., \& Zhou, Y. J. (2015). A dynamical consensus method based on exit-delegation mechanism for large group emergency decision making. KnowledgeBased Systems, 86, 237-249. https://doi.org/10.1016/j.knosys.2015.06.006

$\mathrm{Xu}, \mathrm{Z}$. S. (2005). Deviation measures of linguistic preference relations in group decision making. Omega, 33(3), 249-254. https://doi.org/10.1016/j.omega.2004.04.008

$\mathrm{Xu}, \mathrm{Z}$. S. (2009). An automatic approach to reaching consensus in multiple attribute group decision making. Computers \& Industrial Engineering, 56(4), 1369-1374. https://doi.org/10.1016/j.cie.2008.08.013

Zadeh, L. A. (1965). Fuzzy sets. Information \& Control, 8(3), 338353. https://doi.org/10.1016/S0019-9958(65)90241-X 